\title{
A Method for Separating the Physics from the Astrophysics of High-Redshift 21 Centimeter Fluctuations
}

\section{Citation}

Barkana, Rennan, and Abraham Loeb. 2005. "A Method for Separating the Physics from the Astrophysics of High-Redshift 21 Centimeter Fluctuations." The Astrophysical Journal 624 (2): L65-68. https://doi.org/10.1086/430599.

\section{Permanent link}

http://nrs.harvard.edu/urn-3:HUL.InstRepos:41393417

\section{Terms of Use}

This article was downloaded from Harvard University's DASH repository, and is made available under the terms and conditions applicable to Other Posted Material, as set forth at http:// nrs.harvard.edu/urn-3:HUL.InstRepos:dash.current.terms-of-use\#LAA

\section{Share Your Story}

The Harvard community has made this article openly available. Please share how this access benefits you. Submit a story.

Accessibility 
DRAFT VERSION SEPTEMBER 29, 2018

Preprint typeset using $\mathrm{LT}_{\mathrm{E}} \mathrm{X}$ style emulateapj v. 6/22/04

\title{
A METHOD FOR SEPARATING THE PHYSICS FROM THE ASTROPHYSICS OF HIGH-REDSHIFT 21CM FLUCTUATIONS
}

\author{
RENNAN BARKANA ${ }^{1} \&$ ABRAHAM LOEB ${ }^{2}$ \\ Draft version September 29, 2018
}

\begin{abstract}
Fluctuations in the $21 \mathrm{~cm}$ brightness from cosmic hydrogen at redshifts $z \gtrsim 6$ were sourced by the primordial density perturbations from inflation as well as by the radiation from galaxies. We propose a method to separate these components based on the angular dependence of the $21 \mathrm{~cm}$ fluctuation power spectrum. Peculiar velocities increase the power spectrum by a factor of $\sim 2$ compared to density fluctuations alone, and introduce an angular dependence in Fourier space. The resulting angular structure relative to the line of sight facilitates a simple separation of the power spectrum into several components, permitting an unambiguous determination of the primordial power spectrum of density fluctuations, and of the detailed properties of all astrophysical sources of $21 \mathrm{~cm}$ fluctuations. We also demonstrate that there is no significant information to be gained from $21 \mathrm{~cm}$ measurements on large angular scales. Thus, observational analyses can be confined to small angular areas where projection effects are negligible, and theoretical predictions should focus on the three-dimensional power spectrum of $21 \mathrm{~cm}$ fluctuations.
\end{abstract}

Subject headings: galaxies: high-redshift, cosmology: theory, galaxies: formation

\section{INTRODUCTION}

Following the recombination of protons and electrons less than a million years after the big bang, the universe was filled with neutral hydrogen ( $\mathrm{H} \mathrm{I}$ ). Hundreds of millions of years later, the first galaxies began to reionize the cosmic gas (Barkana \& Loeb 2001). The spectra of the farthest quasars indicate that reionization completed at a redshift $z \sim 6$, a billion years after the big bang (Fan et al. 2002; White et al. 2003; Wvithe et al. 2004). The hyperfine spin-flip transition of $\mathrm{H} \mathrm{I}$ at a wavelength of $21 \mathrm{~cm}$ provides the most promising tracer of the cosmic gas before the end of reionization. Several groups are currently constructing low-frequency radio arrays capable of detecting the diffuse $21 \mathrm{~cm}$ radiation (http://space.mit.edu/eor-workshop/). Since this radiation interacts with $\mathrm{H}$ I through a resonant transition, any observed wavelength selects a redshift slice of the universe. Future measurements of the redshifted $21 \mathrm{~cm}$ brightness as a function of wavelength and direction should provide a threedimensional map of the cosmic H I (Hogan \& Rees 1979; Madau et al. 1997). Fluctuations in the $21 \mathrm{~cm}$ brightness are sourced by primordial density inhomogeneities on all scales down to the cosmological Jeans mass, making $21 \mathrm{~cm}$ the richest cosmological data set on the sky (Loeb \& Zaldarriaga 2004).

The $21 \mathrm{~cm}$ signal can be seen from epochs during which the gas was largely neutral and deviated from thermal equilibrium with the cosmic microwave background (CMB). The signal vanished at redshifts $z \gtrsim 200$, when the residual fraction of free electrons after cosmological recombination kept the gas kinetic temperature, $T_{k}$, close to the CMB temperature, $T_{\gamma}$. But during $200 \gtrsim z \gtrsim 30$ the gas cooled adiabatically and atomic collisions kept the spin temperature of the hyperfine level population below $T_{\gamma}$, so that the gas appeared in absorption (Scott \& Rees 1990; Loeb \& Zaldarriaga 2004).

\footnotetext{
1 School of Physics and Astronomy, The Raymond and Beverly Sackler Faculty of Exact Sciences, Tel Aviv University, Tel Aviv 69978, ISRAEL; barkana@wise.tau.ac.il

2 Astronomy Department, Harvard University, 60 Garden Street, Cambridge, MA 02138; aloeb@cfa.harvard.edu
}

As the Hubble expansion continued to rarefy the gas, radiative coupling of $T_{s}$ to $T_{\gamma}$ began to dominate and the $21 \mathrm{~cm}$ signal faded. When the first galaxies formed, the UV photons they produced between the Ly $\alpha$ and Lyman limit wavelengths propagated freely through the universe, redshifted into the Ly $\alpha$ resonance, and coupled $T_{s}$ and $T_{k}$ once again through the Wouthuysen-Field (Wouthuysen 1952; Field 1958) effect by which the two hyperfine states are mixed through the absorption and re-emission of a Ly $\alpha$ photon (Madau et al. 1997; Ciardi \& Madau 2003). Emission of UV photons above the Lyman limit by the same galaxies initiated the process of reionization by creating ionized bubbles in the neutral cosmic gas, while X-ray photons propagated farther and heated $T_{k}$ above $T_{\gamma}$ throughout the universe. Once $T_{s}$ grew larger than $T_{\gamma}$, the gas appeared in $21 \mathrm{~cm}$ emission. The ionized bubbles imprinted a knee in the power spectrum of $21 \mathrm{~cm}$ fluctuations (Zaldarriaga et al. 2004), which traced the H I topology until the process of reionization was completed (Furlanetto et al. 2004a).

The various effects that determine the $21 \mathrm{~cm}$ fluctuations can be separated into two classes. On the one hand, the density power spectrum probes basic cosmological parameters and inflationary initial conditions, and can be calculated exactly in linear theory. On the other hand, the radiation from galaxies, both $\operatorname{Ly} \alpha$ radiation and ionizing photons, involves the complex, non-linear physics of galaxy formation and star formation. If only the sum of all fluctuations could be measured, then it would be difficult to extract the separate sources, and in particular, the extraction of the power spectrum would be subject to systematic errors involving the properties of galaxies. We show in this Letter that the unique threedimensional properties of $21 \mathrm{~cm}$ measurements permit a separation of these distinct effects. Thus, $21 \mathrm{~cm}$ fluctuations can probe astrophysical (radiative) sources during the epoch of first galaxies, while at the same time separately probing physical (inflationary) sources. In order to affect this separation most easily, it is necessary to measure the three-dimensional power spectrum of $21 \mathrm{~cm}$ fluctuations, while full-sky observations are normally restricted to angular projections. However, we show that unlike the analogous case of CMB fluctuations, 
$21 \mathrm{~cm}$ measurements on large angular scales are not useful. Thus, observational analyses can be confined to small angular areas where projection effects are negligible.

\section{SPIN TEMPERATURE HISTORY}

The spin temperature $T_{S}$ is defined through the ratio between the number densities of hydrogen atoms in the excited and ground state levels, $n_{1} / n_{0}=\left(g_{1} / g_{0}\right) \exp \left\{-T_{\star} / T_{s}\right\}$, where subscripts 1 and 0 correspond to the excited and ground state levels of the $21 \mathrm{~cm}$ transition, $\left(g_{1} / g_{0}\right)=3$ is the ratio of the spin degeneracy factors of the levels, and $T_{\star}=0.0682 \mathrm{~K}$ corresponds to the energy difference between the levels. As long as $T_{s}$ is smaller than the CMB temperature $T_{\gamma}=2.725(1+z) \mathrm{K}$, hydrogen atoms absorb the $\mathrm{CMB}$, whereas if $T_{s}>T_{\gamma}$ they emit excess flux. In general, the resonant $21 \mathrm{~cm}$ interaction changes the brightness temperature of the CMB by (Scott \& Rees 1990; Madau et al. 1997) $T_{b}=\tau\left(T_{s}-T_{\gamma}\right) /(1+z)$, where the optical depth at a wavelength $\lambda=21 \mathrm{~cm}$ is

$$
\tau=\frac{3 c \lambda^{2} h A_{10} n_{\mathrm{H}}}{32 \pi k_{B} T_{s}(1+z)\left(d v_{r} / d r\right)} x_{\mathrm{HI}},
$$

where $n_{H}$ is the number density of hydrogen, $A_{10}=2.85 \times$ $10^{-15} \mathrm{~s}^{-1}$ is the spontaneous emission coefficient, $x_{\mathrm{HI}}$ is the neutral hydrogen fraction, and $d v_{r} / d r$ is the gradient of the radial velocity along the line of sight with $v_{r}$ being the physical radial velocity and $r$ the comoving distance; on average $d v_{r} / d r=H(z) /(1+z)$ where $H$ is the Hubble parameter. The velocity gradient term arises since the $21 \mathrm{~cm}$ scattering crosssection has a fixed thermal width which translates through the redshift factor $\left(1+v_{r} / c\right)$ to a fixed interval in velocity (Sobolev 1960).

For the concordance set of cosmological parameters (Spergel et al. 2003), the mean brightness temperature on the sky at redshift $z$ is $T_{b}=28 \mathrm{mK}[(1+z) / 10]^{1 / 2}\left[\left(T_{s}-T_{\gamma}\right) / T_{s}\right] \bar{x}_{\mathrm{HI}}$, where $\bar{x}_{\mathrm{HI}}$ is the mean neutral fraction of hydrogen. The spin temperature itself is coupled to $T_{k}$ through the spin-flip transition, which can be excited by collisions or by the absorption of Ly $\alpha$ photons. As a result, the combination that appears in $T_{b}$ becomes (Field 1958) $\left(T_{s}-T_{\gamma}\right) / T_{s}=\left[x_{\mathrm{tot}} /(1+\right.$ $\left.\left.x_{\text {tot }}\right)\right]\left(1-T_{\gamma} / T_{k}\right)$, where $x_{\text {tot }}=x_{\alpha}+x_{c}$ is the sum of the radiative and collisional threshold parameters. These parameters are $x_{\alpha}=4 P_{\alpha} T_{\star} / 27 A_{10} T_{\gamma}$ and $x_{c}=4 \kappa_{1-0}\left(T_{k}\right) n_{H} T_{\star} / 3 A_{10} T_{\gamma}$, where $P_{\alpha}$ is the Ly $\alpha$ scattering rate which is proportional to the Ly $\alpha$ intensity, and $\kappa_{1-0}$ is tabulated as a function of $T_{k}$ (Allison \& Dalgarno 1969; Zygelman 2004). The coupling of the spin temperature to the gas temperature becomes substantial when $x_{\text {tot }} \gtrsim 1$.

\section{BRIGHTNESS TEMPERATURE FLUCTUATIONS}

Although the mean $21 \mathrm{~cm}$ emission or absorption is difficult to measure due to bright foregrounds, the unique character of the fluctuations in $T_{b}$ allows for a much easier extraction of the signal (Gnedin \& Shaver 2003; Zaldarriaga et al. 2004; Morales \& Hewitt 2004; Morales 2004; Santos et al. 2004). In general, the fluctuations in $T_{b}$ can be sourced by fluctuations in gas density, temperature, neutral fraction, radial velocity gradient, and Ly $\alpha$ flux (through $x_{\alpha}$ ). Adopting the notation $\delta_{A}$ for the fractional fluctuation in quantity $A$ (with a lone $\delta$ denoting density perturbations), we find

$$
\begin{aligned}
\delta_{T_{b}}= & \left(1+\frac{x_{c}}{\tilde{x}_{\mathrm{tot}}}\right) \delta+\frac{x_{\alpha}}{\tilde{x}_{\mathrm{tot}}} \delta_{x_{\alpha}}+\delta_{x_{\mathrm{HI}}}-\delta_{d_{r} v_{r}} \\
& +\left(\gamma_{a}-1\right)\left[\frac{T_{\gamma}}{T_{k}-T_{\gamma}}+\frac{x_{c}}{\tilde{x}_{\mathrm{tot}}} \frac{d \log \left(\kappa_{1-0}\right)}{d \log \left(T_{k}\right)}\right] \delta,
\end{aligned}
$$

where the adiabatic index is $\gamma_{a}=1+\left(\delta_{T_{k}} / \delta\right)$, and we define $\tilde{x}_{\text {tot }} \equiv\left(1+x_{\text {tot }}\right) x_{\text {tot }}$. Taking the Fourier transform, we obtain the power spectrum of each quantity; e.g., the total power spectrum $P_{T_{b}}$ is defined by

$$
\left\langle\tilde{\delta}_{T_{b}}\left(\mathbf{k}_{1}\right) \tilde{\delta}_{T_{b}}\left(\mathbf{k}_{2}\right)\right\rangle=(2 \pi)^{3} \delta^{D}\left(\mathbf{k}_{1}+\mathbf{k}_{2}\right) P_{T_{b}}\left(\mathbf{k}_{1}\right),
$$

where $\tilde{\delta}_{T_{b}}(\mathbf{k})$ is the Fourier transform of $\delta_{T_{b}}$, $\mathbf{k}$ is the comoving wavevector, and $\langle\cdots\rangle$ denotes an ensemble average.

\section{THE SEPARATION OF POWERS}

The fluctuation $\delta_{T_{b}}$ consists of a number of isotropic sources of fluctuations plus the peculiar velocity term $-\delta_{d_{r} v_{r}}$. Its Fourier transform is simply proportional to that of the density field (Kaiser 1987; Bharadwai \& Ali 2004a),

$$
\tilde{\delta}_{d_{r} v_{r}}=-\mu^{2} \tilde{\delta},
$$

where $\mu=\cos \theta_{k}$ in terms of the angle $\theta_{k}$ of $\mathbf{k}$ with respect to the line of sight. The $\mu^{2}$ dependence in this equation results from taking the radial component $(\propto \mu)$ of the peculiar velocity, and then the radial component $(\propto \mu)$ of its gradient. Intuitively, a high-density region possesses a velocity infall towards the density peak, implying that a photon must travel further from the peak in order to reach a fixed relative redshift, compared with the case of pure Hubble expansion. Thus the optical depth is always increased by this effect in regions with $\delta>0$. This phenomenon is most properly termed velocity compression.

We therefore write the fluctuation in Fourier space as

$$
\tilde{\delta}_{T_{b}}(\mathbf{k})=\mu^{2} \tilde{\delta}(\mathbf{k})+\beta \tilde{\delta}(\mathbf{k})+\tilde{\delta}_{\text {rad }}(\mathbf{k}),
$$

where we have defined a coefficient $\beta$ by collecting all terms $\propto \delta$ in equation (2), and have also combined the terms that depend on the radiation fields of Ly $\alpha$ photons and ionizing photons, respectively. We assume that these radiation fields produce isotropic power spectra, since the physical processes that determine them have no preferred direction in space. The total power spectrum is

$$
\begin{aligned}
P_{T_{b}}(\mathbf{k})= & \mu^{4} P_{\delta}(k)+2 \mu^{2}\left[\beta P_{\delta}(k)+P_{\delta-\mathrm{rad}}(k)\right]+ \\
& {\left[\beta^{2} P_{\delta}(k)+P_{\mathrm{rad}}(k)+2 \beta P_{\delta-\mathrm{rad}}(k)\right], }
\end{aligned}
$$

where we have defined the power spectrum $P_{\delta-\text { rad }}$ as the Fourier transform of the cross-correlation function,

$$
\xi_{\delta-\operatorname{rad}}(r)=\left\langle\delta\left(\mathbf{r}_{1}\right) \delta_{\text {rad }}\left(\mathbf{r}_{1}+\mathbf{r}\right)\right\rangle .
$$

We note that a similar anisotropy in the power spectrum has been previously derived in a different context, that of measuring density fluctuations from galaxy redshift surveys. In that case, the use of redshifts to estimate distances changes the apparent density of galaxies along the line of sight (Kaiser 1987; Lilie \& Efstathiou 1989; Hamilton 1992; Fisher, Scharf, \& Lahav 1994). However, in the case of galaxies there is no analog to the method that we demonstrate below for separating in $21 \mathrm{~cm}$ fluctuations the effect of initial conditions from that of later astrophysical processes.

We may now calculate the angular power spectrum of the brightness temperature on the sky at a given redshift - corresponding to a comoving distance $r_{0}$ along the line of sight. The brightness fluctuations can be expanded in spherical harmonics with expansion coefficients $a_{l m}(\nu)$, where the angular power spectrum

$$
\begin{aligned}
C_{l}\left(r_{0}\right)= & \left\langle\left|a_{l m}(\nu)\right|^{2}\right\rangle=4 \pi \int \frac{d^{3} k}{(2 \pi)^{3}}\left[G_{l}^{2}\left(k r_{0}\right) P_{\delta}(k)+\right. \\
& \left.2 P_{\delta-\operatorname{rad}}(k) G_{l}\left(k r_{0}\right) j_{l}\left(k r_{0}\right)+P_{\mathrm{rad}}(k) j_{l}^{2}\left(k r_{0}\right)\right],
\end{aligned}
$$


with $G_{l}(x) \equiv J_{l}(x)+(\beta-1) j_{l}(x)$ and $J_{l}(x)$ being a linear combination of spherical Bessel functions (Bharadwai \& Ali 2004a).

The velocity gradient term has been previously neglected in $21 \mathrm{~cm}$ calculations, except for its effect on the skyaveraged power and on radio visibilities (Tozzi et al. 2000; Bharadwai \& Ali 2004a b). In the simple case where $\beta=1$ and only the density and velocity terms contribute, the velocity term increases the total power by a factor of $\left\langle\left(1+\mu^{2}\right)^{2}\right\rangle=$ 1.87 in the spherical average. However, instead of averaging the signal, we can use the angular structure of the power spectrum to greatly increase the discriminatory power of $21 \mathrm{~cm}$ observations. We may break up each spherical shell in $\mathbf{k}$ space into rings of constant $\mu$ and construct the observed $P_{T_{b}}(k, \mu)$. Considering equation (6) as a polynomial in $\mu$, i.e., $\mu^{4} P_{\mu^{4}}+\mu^{2} P_{\mu^{2}}+P_{\mu^{0}}$, we see that the power at just three values of $\mu$ is required in order to separate out the coefficients of 1 , $\mu^{2}$, and $\mu^{4}$ for each $k$.

If the velocity compression were not present, then only the $\mu$-independent term (times $T_{b}^{2}$ ) would be observed, and its separation into the five components $\left(T_{b}, \beta\right.$, and three power spectra) would be difficult and subject to degeneracies. Once the power has been separated into three parts, however, the $\mu^{4}$ coefficient can be used to measure the density power spectrum directly, with no interference from any other source of fluctuations. Since the overall amplitude of the power spectrum, and its scaling with redshift, are well determined from the combination of the CMB temperature fluctuations and galaxy surveys, the amplitude of $P_{\mu^{4}}$ directly determines the mean brightness temperature $T_{b}$ on the sky, which measures a combination of $T_{s}$ and $\bar{x}_{\mathrm{HI}}$ at the observed redshift. Once $P_{\delta}(k)$ has been determined, the coefficients of the $\mu^{2}$ term and the $\mu$-independent term must be used to determine the remaining unknowns, $\beta, P_{\delta-\text { rad }}(k)$, and $P_{\text {rad }}(k)$. Since the coefficient $\beta$ is independent of $k$, determining it and thus breaking the last remaining degeneracy requires only a weak additional assumption on the behavior of the power spectra, such as their asymptotic behavior at large or small scales. If the measurements cover $N_{k}$ values of wavenumber $k$, then one wishes to determine $2 N_{k}+1$ quantities based on $2 N_{k}$ measurements, which should not cause significant degeneracies when $N_{k} \gg 1$. Even without knowing $\beta$, one can probe whether some sources of $P_{\mathrm{rad}}(k)$ are uncorrelated with $\delta$; the quantity $P_{\text {un }-\delta}(k) \equiv P_{\mu^{0}}-P_{\mu^{2}}^{2} /\left(4 P_{\mu^{4}}\right)$ equals $P_{\text {rad }}-P_{\delta \text {-rad }}^{2} / P_{\delta}$, which receives no contribution from any source that is a linear functional of the density distribution.

\section{SPECIFIC EPOCHS}

At $z \sim 35$, galaxy formation is in its infancy, while collisions are effective due to the high gas density (Loeb \& Zaldarriaga 2004). Thus, $x_{\mathrm{tot}}=x_{c} \gg x_{\alpha}, x_{\mathrm{HI}}=1$, and the gas cools adiabatically (with $\gamma_{a}=5 / 3$ ). In addition to measuring the density power spectrum(Loeb \& Zaldarriaga 2004), one can also trace the redshift evolution of $n_{\mathrm{HI}}, T_{\gamma}$, and $T_{k}$, and verify that collisions are causing the fluctuations.

At $z \lesssim 35$, collisions become ineffective but the first stars produce a cosmic background of Ly $\alpha$ photons that couples $T_{s}$ to $T_{k}$. During the period of initial Ly $\alpha$ coupling, fluctuations in the $\operatorname{Ly} \alpha$ flux translate into fluctuations in the $21 \mathrm{~cm}$ brightness (Barkana \& Loeb 2004). This signal can be observed from $z \sim 25$ until the Ly $\alpha$ coupling is completed (i.e., $x_{\mathrm{tot}} \gg 1$ ) at $z \sim 15$. At a given redshift, each atom sees Ly $\alpha$ photons that were originally emitted at earlier times at rest-frame wavelengths between Ly $\alpha$ and the Lyman limit. Distant sources

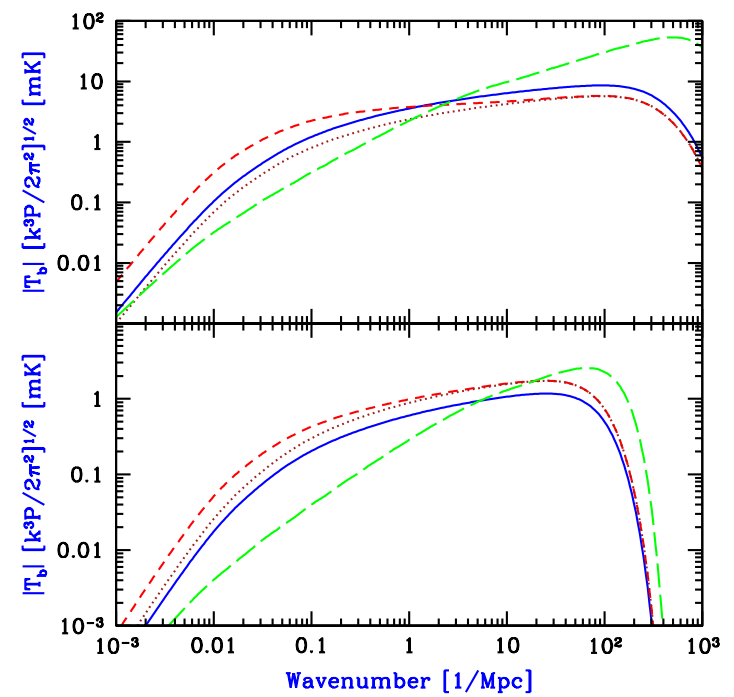

FIG. 1.- Observable power spectra during the period of initial Ly $\alpha$ coupling. Upper panel: Assumes adiabatic cooling. Lower panel: Assumes pre-heating to $500 \mathrm{~K}$ by X-ray sources. We show $P_{\mu^{4}}=P_{\delta}$ (solid curves), $P_{\mu^{2}}$ (short-dashed curves), and $P_{\mathrm{un}-\delta}$ (long-dashed curves). We also show for comparison $2 \beta P_{\delta}$ (dotted curves).

are time retarded, and since there are fewer galaxies in the distant, earlier universe, each atom sees sources only out to an apparent source horizon of $\sim 100$ comoving Mpc at $z \sim 20$. A significant portion of the flux comes from nearby sources, because of the $1 / r^{2}$ decline of flux with distance, and since higher Lyman series photons, which are degraded to Ly $\alpha$ photons through scattering, can only be seen from a small redshift interval that corresponds to the wavelength interval between two consecutive atomic levels.

There are two separate sources of fluctuations in the Ly $\alpha$ flux (Barkana \& Loeb 2004). The first is density inhomogeneities. Since gravitational instability proceeds faster in overdense regions, the biased distribution of rare galactic halos fluctuates much more than the global dark matter density. When the number of sources seen by each atom is relatively small, Poisson fluctuations provide a second source of fluctuations. Unlike typical Poisson noise, these fluctuations are correlated between gas elements at different places, since two nearby elements see many of the same sources. Assuming a scale-invariant spectrum of primordial density fluctuations, and that $x_{\alpha}=1$ is produced at $z=20$ by galaxies in dark matter halos where the gas cools efficiently via atomic cooling, we show in Figure 1 the predicted observable power spectra. The figure shows that $\beta$ can be measured from the ratio $P_{\mu^{2}} / P_{\mu^{4}}$ at $k \gtrsim 1 \mathrm{Mpc}^{-1}$, allowing the density-induced fluctuations in flux to be extracted from $P_{\mu^{2}}$, while only the Poisson fluctuations contribute to $P_{\mathrm{un}-\delta}$. Each of these components probes the number density of galaxies through its magnitude, and the distribution of source distances through its shape. Measurements at $k \gtrsim 100 \mathrm{Mpc}^{-1}$ can independently probe $T_{k}$ because of the smoothing effects of the gas pressure and the thermal width of the $21 \mathrm{~cm}$ line.

After Ly $\alpha$ coupling and X-ray heating are both completed, reionization continues. Since $\beta=1$ and $T_{k} \gg T_{\gamma}$, the normalization of $P_{\mu^{4}}$ directly measures the mean neutral hydrogen fraction, and one can separately probe the density fluctuations, the neutral hydrogen fluctuations, and their cross- 


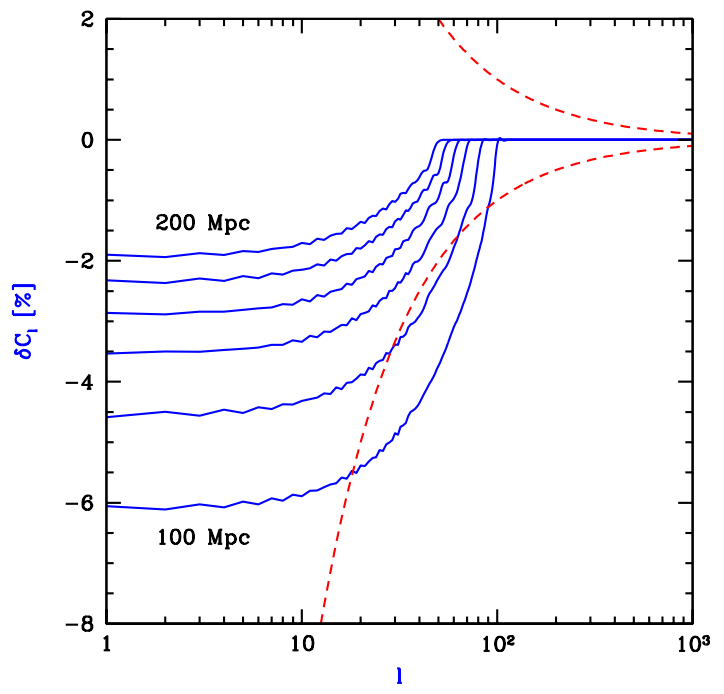

FIG. 2.- Effect of large-scale power on the angular power spectrum of $21 \mathrm{~cm}$ anisotropies on the sky. This example shows the power from density fluctuations and velocity compression, assuming a hot IGM at $z=12$ with $T_{s}=T_{k} \gg T_{\gamma}$. We show the \% change in $C_{l}$ if we were to cut off the power spectrum above $1 / k$ of $200,180,160,140,120$, and $100 \mathrm{Mpc}$ (top to bottom). Also shown for comparison is the cosmic variance for averaging in bands of $\Delta l \sim l$ (dashed lines).

correlation.

\section{FLUCTUATIONS ON LARGE ANGULAR SCALES}

Full-sky observations must normally be analyzed with an angular and radial transform, rather than a Fourier transform which is simpler and yields more directly the underlying 3D power spectrum. In an angular transform on the sky, an angle of $\theta$ radians translates to a spherical multipole $l \sim 3.5 / \theta$. For measurements on a screen at a comoving distance $r_{0}$, a multipole $l$ normally measures 3D power on a scale of $k^{-1} \sim \theta r_{0} \sim 35 / l \mathrm{Gpc}$ for $l \gg 1$, since $r_{0} \sim 10 \mathrm{Gpc}$ at $z \gtrsim 10$. This estimate fails at $l \lesssim 100$, however, when we consider the sources of $21 \mathrm{~cm}$ fluctuations. The angular projection implied in $C_{l}$ involves a weighted average [equation [8] which favors large scales when $l$ is small, but density fluctuations possess little large-scale power, and the $C_{l}$ are dominated by power around the peak of $k P_{\delta}(k)$, at a few tens of comoving Mpc.

Figure 2 shows that for density and velocity fluctuations, even the $l=1$ multipole is affected by power at $k^{-1}>200 \mathrm{Mpc}$ only at the $2 \%$ level. Due to the small number of large angular modes available on the sky, the expectation value of $C_{l}$ cannot be measured precisely at small $l$. Figure 2 shows that this precludes new information from being obtained on scales $k^{-1} \gtrsim 130 \mathrm{Mpc}$ using angular structure at any given redshift. An optimal observing strategy would be to divide the sky into separate angular pixels of size $\theta \lesssim 2^{\circ}$, in which the curvature changes distances by only $\theta^{2} / 24 \sim 7 \times 10^{-5}$. This would allow a direct 3D Fourier transform, which is also the most natural way of analyzing radio observations (Morales \& Hewitt 2004; Morales 2004).

\section{SUMMARY}

We have argued that observers should try to measure the anisotropic form of the power spectrum of $21 \mathrm{~cm}$ fluctuations. Since measurements on large angular scales are not worthwhile, the three-dimensional power spectrum may indeed be measured by dividing the sky into small angular areas. The anisotropy itself, which is due to the effect of peculiar velocities, facilitates a simple separation of the power spectrum into three separate components. One component allows an unambiguous determination of the primordial power spectrum of gas density fluctuations, which if measured will probe basic cosmological parameters as well as inflation. The other two components probe the properties of astrophysical (radiative) sources of $21 \mathrm{~cm}$ fluctuations.

This work was supported in part by NSF grants AST0204514, AST-0071019 and NASA grant NAG 5-13292 . R.B. is grateful for the kind hospitality of the HarvardSmithsonian CfA, and acknowledges the support of an Alon Fellowship at Tel Aviv University and of Israel Science Foundation grant 28/02/01.

\section{REFERENCES}

Allison, A. C., \& Dalgarno, A. 1969, ApJ, 158, 423

Barkana, R., \& Loeb, A. 2001, Phys. Rep., 349, 125

Barkana, R., \& Loeb, A. 2004, ApJ, submitted.

Bharadwaj, S., \& Ali, S. S. 2004a, MNRAS, 352, 142

Bharadwaj, S., \& Ali, S. S. 2004b, MNRAS, submitted, preprint astro-ph/0406676

Ciardi, B. \& Madau, P. 2003, ApJ, 596, 1

Fan, X., et al. 2002, AJ, 123, 1247

Field, G. B. 1958, Proc. IRE, 46, 240

Fisher, K. B., Scharf, C. A., \& Lahav, O. 1994, MNRAS, 266, 219

Furlanetto, S. R., Zaldarriaga, M. \& Hernquist, L. 2004a, ApJ, submitted, preprint astro-ph/0403697

Gnedin, N. Y., \& Shaver, P. A. 2003, ApJ, submitted, preprint astro-ph/0312005

Hamilton, A. 1992, ApJ, 385, L5

Hogan, C. J., \& Rees, M. J. 1979, MNRAS, 188, 791

Kaiser, N. 1987, MNRAS, 227, 1

Lilje, P. B., \& Efstathiou, G. 1989, MNRAS, 236, 851
Loeb, A., \& Zaldarriaga, M. 2004, Phys. Rev. Lett., 92, 211301

Madau, P., Meiksin, A., \& Rees, M. J. 1997, ApJ, 475, 429

Morales, M. F., ApJ, submitted, preprint astro-ph/0406662

Morales, M. F., \& Hewitt, J., ApJ, in press, preprint astro-ph/0312437

Santos, M. G., Cooray, A., \& Knox, L. 2004, preprint astro-ph/0408515

Scott, D. \& Rees, M. J. 1990, MNRAS, 247, 510

Sobolev, V. V. 1960, Moving Envelopes of Stars, Cambridge: Harvard University Press

Spergel, D. N., et al. 2003, ApJS, 148, 175

Tozzi, P., Madau, P., Meiksin, A., \& Rees, M. J. 2000, ApJ, 528, 597

White, R. L., Becker, R.H., Fan, X., \& Strauss, M.A. 2003, AJ, 126, 1

Wouthuysen, S. A., 1952, AJ, 57, 31

Wyithe, J.S.B., \& Loeb, A. 2004, Nature, 427, 815

Zaldarriaga, M., Furlanetto, S. R., \& Hernquist, L. 2004, ApJ, 608, 622

Zygelman, B. 2004, in preparation 